\title{
Chronic fatigue syndrome following typhoid infection: neurasthenia
}

\begin{abstract}
Chronic fatigue syndrome is often the least diagnosable psychiatric illness. It mostly presents to the physician with vague symptoms. The usual presentation is characterised by profound fatigue, sleep abnormalities, pain and other symptoms that are made worse by exertion. There are no medical causes for this illness however all of them have to be excluded to come to a diagnosis of CFS. It may affect women more than males and maybe associated with some viral or bacterial infections etc but usually all investigations are normal. There is no specific treatment for this condition.
\end{abstract}

Keywords: chronic fatigue syndrome, typhoid, hypothyroidism, weakness, tiredness
Volume 9 Issue I - 2019

\author{
Amitabh Saha,VV Gantait \\ Department of Psychiatry, India
}

Correspondence: Dr. Amitabh Saha, MD Psychiatry, Department of Psychiatry, Kolkata, India,

Email sahaing@gmail.com

Received: October 15, 2018| Published: January 09, 2019

\section{Introduction}

Chronic fatigue syndrome is the diagnosis of exclusion. To the untrained eye this condition is often missed. Chronic fatigue syndrome (CFS) is usually a chronic and debilitating disorder which is often characterized by extreme fatigue or tiredness that doesn't go away with rest and can't be explained by an underlying medical condition. This illness at times is also named as myalgic encephalomyelitis (ME) or systemic exertion intolerance disease (SEID). ${ }^{1}$ The etiology of CFS is not very well well understood even with the advent of all newer modalities of investigations. ${ }^{2}$ Some theories include viral illness, stress or a mix of both however as there are no specific tell tale signs of a single cause it becomes difficult to find out the cause.

There are no laboratory tests or imaging studies or CSF studies for CFS, so the treating doctor will have to negate out all other causes for the tiredness or fatigue when figuring out a diagnosis. ${ }^{3}$ It's now being widely being seen as a medical condition however psychiatrists are also diagnosing them and providing some form of treatment.

Chronic Fatigue Syndrome can affect anyone, though it's most common among middle aged women in their 40s and 50s. There's currently no cure, so treatment for CFS focuses on relieving symptom or symptom reduction.

The cause of CFS is unknown. Researchers speculate that contributing factors may include the following

- Bacterial infection: which may include Coxiella

burnetii and mycoplasma pneumoniae, etc also been studied in relation to CFS.

- Viruses: example includes Epstein-Barr virus (EBV), human herpes virus 6, Ross River virus (RRV), rubella

- a compromised immune system

- stress

- hormonal disturbances

- Genetics; some are genetically predisposed to develop CFS.

CFS can be the end stage of multiple different conditions, rather than one unique condition. In fact, around 1 in 10 people with EpsteinBarr virus, Ross River virus, and Coxiella burnetii develop a condition that meets the criteria for a CFS diagnosis. Additionally, they say that those who've had severe symptoms with any of these three infections are at a higher risk of later developing chronic fatigue syndrome.

\section{Risk factors for CFS}

CFS is most commonly seen among people in their 40 s and 50 s. Gender also plays an important role in CFS, as women are 2:4> males predisposed more likely to be diagnosed with CFS than men. ${ }^{4}$ Genetic predisposition, allergies, stress, and environmental factors may also increase the risk. ${ }^{5}$

\section{Symptoms}

The symptoms of CFS vary based on the individual affected and the severity of the condition seen in such cases. The most common symptom is tiredness or fatigue that's severe enough to interfere with persons daily activities or routine work.. For CFS to be diagnosed, fatigue must last for at least six months and must not be curable with bed rest or lying down. ${ }^{6}$

Other symptoms of CFS may include:

- severe fatigue that's not improved by rest

- post-exertional malaise (PEM), where your symptoms get worse after any physical or mental activity

- reduced or loss of memory or concentration

- feeling unrefreshed after a night's sleep or feeling lazy on getting up

- chronic insomnia (and other sleep disorders)

- muscle pain

- frequent headaches

- multi-joint pain without redness or swelling

- frequent sore throat

- tender and swollen lymph nodes in your neck and armpits

\section{Treatment}

Making some subtle changes to a person's lifestyle can help reduce the symptoms. Limiting or eliminating your caffeine intake will help him to sleep better and ease his sleep disturbance or insomnia. The limiting of nicotine and alcohol intake, too will help in reducing the severity of illness. To avoid napping during the day if it's hurting his ability to sleep at night can also be tried out .Sleep hygiene and creating a sleep routine. Going to bed at the same time every night and aim to wake up around the same time every day goes a long way to help the person. ${ }^{7}$ 
Medications:- Typically, no one medication can treat all of your symptoms. Also, your symptoms may change over time, so your medications may have to change also .In many cases, CFS can trigger or be a part of depression, and you may need an antidepressant for it.If lifestyle changes don't give you a restful night's sleep, hence the doctor may suggest a sleep aid. Pain-reducing medication can also help you cope with aches and joint pain caused by CFS.

\section{Alternative medicine}

Measure like - Acupuncture, tai chi, yoga, and massage may help relieve the pain associated with $\mathrm{CFS}{ }^{8}$

\section{Case report}

\section{Reason for referral}

This 53 yrs old patient, self reported the outpatient department in Mar 2018 with h/o generalized weakness, reduced appetite, fever on $\&$ off since 03 yrs. Initially he was evaluated by med spl and found to have excessive preoccupation with his somatic complaints, hence psychiatric consultation was sought.

\section{History of illness}

Detailed history revealed that indl was apparently asymptomatic till 2014 when he had a febrile illness lasting for 1 month. He was told by civil doctor that he was having Typhoid fever and gave him some oral medication with which he improved. But he started having lethargy and generalized weakness after recovery. In Nov 2015, while posted at Srinagar, he again had fever with rash which was managed at a local hospital. Following which he started having reduced energy, low mood which was not persistent and pervasive. However he was functioning satisfactorily in his socio-occupational sphere.

In Feb 2018, while on leave, he had developed sleep disturbances in the form of initial insomnia, generalized weakness, heaviness of head and multiple muscle aches and pains. He used to stay largely on bed and had reduced interaction with others. He consulted a civil doctor and during investigations, he was found to have raised TSH. He was diagnosed with Hypothyroidism and started on Thyroxine 50mcg OD. On 03 Mar 2018, he reported back to duty but continued to have generalized body ache, reduced sleep and appetite. He used to be distressed about persistent fatigue and exhaustion. He was finally admitted at a tertiary care centre for persistent symptoms.

No history of persistent pervasive sadness of mood/suspiciousness/ fearfulness/hearing disembodied voices. No h/o head injury/seizure/ fever with altered sensorium.

\section{Past, family history and personal history}

No past history of any significant medical or surgical illness. He hailed from rural agrarian region of Moga distt in Punjab. His father was a labourer in PWD in Moga. Father died in 1995 at the age of 78 yrs due to old age. Mother was a home maker died at the age of 75 yrs due to old age.

Denies history of any Psychiatric illness in any of the family members. Educated upto $5^{\text {th }}$ std. He got enrolled in army in 1984 to earn a livelihood. Joined a job year 2007 owing to financial constraints. Though he wanted to enjoy his retirement but he had to take extension in service to cater for his financial needs. He got married in year 1986. Claims cordial relationship with wife. The couple has 3 daughters and a son. Premorbidly he has neurotic traits.

\section{Condition \& course in hospital}

During his initial evaluation, his general and systemic examination were WNL. MSE revealed him to be a well kempt, co-operative indl, with adequate eye contact, normal PMA and low volume of speech, euthymic affect. He was preoccupied with his decreasing mental and bodily well- being. No depressive cognition/ suicidal ideations/ delusions could be elicited. He had normal perception in clear sensorium. He had intact judgment, adequate insight to his illness and deranged bio-drive in the form of disturbed sleep \& reduced appetite and energy. His initial BDI score was 17 . In view of chronic fatiguability, low mood, reduced sleep and appetite, provisional diagnosis of Dysthymia was considered. He was managed with Tab Mirtazepine $15 \mathrm{mg} / \mathrm{d}$, Tab Dothiepin $75 \mathrm{mg} / \mathrm{d}$, Tab Eltroxin $50 \mathrm{mcg}$ /d. However he continued to have lethargy, reduced appetite and low mood.

He was further evaluated and found to have raised TSH and anti TPO antibodies. Endocrinologist opined that his persistent weakness ca not be explained by his mild Hypothyroid status. His longitudinal ward observation did not reflect any affective psychopathology. He used to participate in ward activities and interacting normally with others.

In view of presence of distressing fatiguibality, frequents body aches, sleep disturbances and absence of any mood symptoms or psychotic symptoms, his diagnosis was modified to Neurasthenia. He was managed with Capfluoxetine, Tab Mirtazepine $7.5 \mathrm{mg} / \mathrm{d}$. He was given benefit of psychoeducation, graded exercises therapy and CBT. He claimed to have improvement in symptoms. Indl was sent on 06 weeks sick leave for convalescence. Sick leave period was uneventful and he remained compliant to medications. Post his leave period on being reviewed, he expressed confidence to continue in service. Serial MSE and ward observation did not reveal any overt psychopathology. He was advised to continue with Tab Mirtazapine7.5mg0-0-1, Cap Fluoxetine 20mg 1-0-0, Tab Eltroxin 50mcg 1-0-0 at time of discharge.

\section{Discussion}

Despite increased research efforts and advanced investigative modalities, CFS remains a poorly understood condition with no absolute or palliative cure in sight with many patients continuing the treatment for a long time. Managing CFS can therefore be challenging to the doctor. ${ }^{9}$ It will require a person to make major lifestyle changes in order to adapt to his chronic fatigue and tiredness. As a result, the person may experience depression, anxiety, or social isolation, so some people find that joining a support group can be helpful. ${ }^{10}$

CFS progresses differently in different people in different culture and in different strata of society, so it's important to work with the doctor to come up with a treatment plan that meets patient needs and demands. Many people benefit from working with a team of healthcare providers, including doctors, therapists, and rehabilitation specialists.${ }^{11}$ If he is living with this condition, the Solve ME/CFS Initiative has resources that he may find helpful. The CDC also offers recommendations for managing and living with CFS. ${ }^{12}$ As it affects a person's mental health and reduces his self confidence and self esteem it is essential that the person seek professional help as early as possible. ${ }^{13}$

\section{Acknowledgments}

None. 


\section{Conflicts of interest}

The author declares no conflicts of interest.

\section{References}

1. Myalgic Encephalomyelitis/Chronic Fatigue Syndrome Symptoms and Diagnosis. CDC. 2017.

2. Chronic fatigue syndrome/myalgic encephalomyelitis (or encephalopathy): diagnosis and management. London: National Institute for Health and Clinical Excellence. 2017.

3. ME/CFS, Myalgic Encephalomyelitis/Chronic Fatigue Syndrome: Advancing the Research; Background; Pathways to Prevention (P2P) Workshop. prevention.nih.gov. 2017.

4. Elgot Jessica. Chronic fatigue patients criticise study that says exercise can help. The Guardian. 2015.

5. Research, Center for Drug Evaluation and. "FDA Response Letter Regarding Approval of Ampligen for ME/CFS". 2018.

6. CDC. Myalgic Encephalomyelitis/Chronic Fatigue Syndrome ME/CFS in Children. Centers for Disease Control and Prevention. 2017.
7. Ottati VC, Tindale RS, Edwards J, et al. The social psychology of politics. New York: Kluwer Academic/Plenum. pp. 159-160.

8. Price JR, Mitchell E, Tidy E, et al. Cognitive behaviour therapy for chronic fatigue syndrome in adults. Cochrane Database Syst Rev. 2008;16(3):CD001027.

9. Brurberg Kjetil Gundro, Fønhus Marita Sporstøl, Larun Lillebeth, et al. Case definitions for chronic fatigue syndrome/myalgic encephalomyelitis (CFS/ME): a systematic review. BMJ Open. 2014;4(2):e003973.

10. Symptoms Myalgic Encephalomyelitis/Chronic Fatigue Syndrome (ME/CFS). 2017.

11. "CDC - Chronic Fatigue Syndrome (CFS) — Diagnosis”. 2012.

12. "CDC, Chronic Fatigue Syndrome (CFS), Making a Diagnosis" (PDF). 2011.

13. Nijs J, Meeus M, Van Oosterwijck J, et al. In the mind or in the brain? Scientific evidence for central sensitisation in chronic fatigue syndrome. Eur J Clin Invest. 2012;42(2): 203-212. 